\title{
A molecular outflow driven by the brown dwarf binary FU Tauri ${ }^{\star}$
}

\author{
J.-L. Monin ${ }^{1}$, E. T. Whelan ${ }^{2}$, B. Lefloch ${ }^{1}$, C. Dougados ${ }^{1}$, and C. Alves de Oliveira ${ }^{3}$ \\ ${ }^{1}$ UJF-Grenoble/CNRS-INSU, Institut de Planétologie et d'Astrophysique de Grenoble (IPAG) UMR 5274, 38041 Grenoble, France \\ e-mail: Jean-Louis. Monin@obs.ujf-grenoble.fr \\ 2 Institut für Astronomie und Astrophysik, Kepler Center for Astro and Particle Physics, Sand 1, Eberhard Karls Universität, \\ 72076 Tübingen, Germany \\ 3 European Space Astronomy Centre (ESA), PO Box 78, 28691 Villanueva de la Cañada, Madrid, Spain
}

Received 20 December 2012 / Accepted 18 January 2013

\section{ABSTRACT}

\begin{abstract}
We report the detection of a molecular outflow driven by the brown dwarf binary FU Tau. Using the IRAM $30 \mathrm{~m}$ telescope we observed the ${ }^{12} \mathrm{CO}(2-1)(\mathrm{CO})$ emission in the vicinity of FU Tau and detected a bipolar outflow by examining the wings of the $\mathrm{CO}(2-1)$ line as we moved away from the source position. An integrated map of the wing emission between $3 \mathrm{~km} \mathrm{~s}^{-1}$ and $5 \mathrm{~km} \mathrm{~s}^{-1}$ reveals a blueshifted lobe at a position of $\sim 20^{\prime \prime}$ from the FU Tau system and at a position angle of $\sim 20^{\circ}$. The beam size of the observations is $11^{\prime \prime}$, so it is not possible to distinguish between the two components of the FU Tau binary. Since optical forbidden emission, a strong tracer of the shocks caused by outflow activity, has been detected in the spectrum of FU Tau A, we assume that this component is the driving source of the molecular outflow. We estimate the mass of the outflow at $4 \times 10^{-6} M_{\odot}$ and the mass outflow rate at $6 \times 10^{-10} M_{\odot} / \mathrm{yr}$. These results agree well with previous estimates for BD molecular outflows. FU Tau A is now the third BD found to be associated with molecular outflow activity, and this discovery adds to the already extensive list of the interesting properties of FU Tau.
\end{abstract}

Key words. radio lines: ISM - brown dwarfs - stars: formation - stars: pre-main sequence - stars: winds, outflows

\section{Introduction}

Young brown dwarfs (BDs) occupy the mass regime between stars and planets and are therefore significant in any theory describing activity in star-forming regions. Thus they have become the subjects of increased scrutiny in recent years (Luhman 2012). Their formation mechanism is at present much debated, and indeed it has been postulated that they may form by more than one mechanism (Whitworth et al. 2006). The simplest idea is that they form in the same manner as low-mass stars, i.e. through the gravitational collapse of substellar mass cores (Padoan \& Nordlund 2004). These cores occur directly by the process of turbulent fragmentation. In this scenario, BDs are just scaled-down versions of low-mass stars. Detailed studies of the circumstellar environments of young BDs provide critical constraints to different formation mechanisms and are needed to identify the dominant mechanism. In particular, if BDs form like low-mass stars, we expect their accretion/outflow properties to be analogous. As a low-mass star forms, it displays a series of ubiquitous observational properties, such as accretion disks, outflows, and excess emission in the near-infrared and visual absorption.

The observational evidence gathered to date in various wavelength domains indicates that young BDs show accretion and ejection behaviour similar to low-mass stars; for example, they demonstrate T Tauri-like accretion (Jayawardhana et al. 2003; Natta et al. 2004; Monin et al. 2010; Rigliaco et al. 2011) and both optical and molecular outflows, driven by BDs, have been detected. ISO-Oph 102 is a good example. It is an accretor with an observed accretion disk (Natta et al. 2002, 2004) and recent ALMA observations have detected millimeter sized grains in its disk (Ricci et al. 2012). Its optical jet was discovered

\footnotetext{
* Based on observations carried out with the IRAM 30 m Telescope. IRAM is supported by INSU/CNRS (France), MPG (Germany), and IGN (Spain).
}

by Whelan et al. (2005) through spectro-astrometric analysis of the $[\mathrm{O}] \lambda 6300$ emission line. Forbidden emission lines (FELs) like [OI] $\lambda 6300$ are important coolants in shocks, hence good tracers of jets. Traditionally, jets from classical T Tauri stars (CTTSs) are investigated by studying their FEL regions. Phan-Bao et al. (2008) also detected a CO molecular outflow driven by ISO-Oph 102. The orientation of the blue and red lobes agreed with the optical observations.

The question of outflow activity in BDs is an important one, as a sufficiently efficient outflow activity could provide an explanation as to why the central object mass does not reach the $\mathrm{H}$ burning limit (Bacciotti et al. 2011; Whelan et al. 2009; Machida et al. 2009). Molecular outflows are an important largescale expression of jet launching. Indeed, molecular outflows were one of the first observational manifestations of this process to be studied (Reipurth \& Bally 2001; Bachiller 1996). While giant Herbig-Haro $(\mathrm{HH})$ flows are optically visible and composed of many $\mathrm{HH}$ objects, where each group represents different episodes of mass ejection, molecular outflows begin when the powerful bipolar jets accelerate and drive the molecular gas in the vicinity of their parent star outwards. Although it is accepted that they are powered by the primary jet from the protostar, the exact way in which the jet interacts with the molecular material is still uncertain (Cabrit et al. 1997; Downes \& Ray 1999; Downes \& Cabrit 2007). Molecular outflows are primarily detected in the $\mathrm{CO}$ molecule, and thus millimeter observations have dominated the search for them. These outflows are mainly detected from Class 0 and I low-mass stars that are still embedded in their natal material. Observations of molecular outflows driven by the more evolved Class II CTTSs are much rarer (Cabrit et al. 2011).

As of today, only two detections of molecular outflows from optically visible young BDs have been made so far (Phan-Bao et al. 2008, 2011), although it is postulated that owing to the 
colder environment of BDs, molecular outflows may be more common than in CTTS. We have conducted a survey of young BDs with the IRAM $30 \mathrm{~m}$ telescope in the ${ }^{12} \mathrm{CO}(1-0)$ and ${ }^{12} \mathrm{CO}(2-1)$ to test this hypothesis (Whelan et al., in prep.). Their approach is to target BDs known to be accreting and that also show evidence of outflow activity primarily in the form of FELs, in a mass range of $0.02 M_{\odot}$ to $0.13 M_{\odot}$, including a few very low-mass stars (VLMSs). In this letter we report the detection of a remarkable molecular outflow in FU Tau, as part of our IRAM survey.

FU Tau $\left(04^{\mathrm{h}} 23^{\mathrm{m}} 35^{\mathrm{s}} 4,+25^{\circ} 03^{\prime} 03^{\prime \prime} 05\right)$ is a BD-BD binary with a projected angular separation of $5 . \prime 7$ or 800 AU at the distance to Taurus and a position angle (PA) of $\sim 145^{\circ}$ (Luhman et al. 2009). Its membership of the Taurus molecular cloud has been known for some time (Jones \& Herbig 1979), and it is situated in a relatively isolated region of the cloud. Luhman et al. (2009) give the spectral type of FU Tau A at M7.25, corresponding to a mass of $50 M_{\mathrm{Jup}}$ and the spectral type and mass of the companion at M9.5 and $15 M_{\text {Jup }}$, respectively. The wide nature of the FU Tau binary challenges models that suggest that BDs form when their accretion is halted owing to ejection from their natal clouds, since the system appears to have formed irrespective of dynamical interaction with nearby stars.

A further intriguing property of FU Tau A is its overluminosity with respect to other members of the Taurus star-forming region of the same spectral type (Luhman et al. 2009; Scholz et al. 2011). The spectral energy distributions (SEDs) of both components show excess emission that indicates the presence of circumstellar disks, and their disks are classified as Class II by Luhman et al. (2009). Furthermore, optical spectra clearly show that accretion is ongoing in FU Tau A. Stelzer et al. (2010) estimate the mass accretion rate from both the $\mathrm{H} \alpha$ and He I ( 25876$)$ lines with $\dot{M}_{\mathrm{H} \alpha}=3.5 \times 10^{-10} M_{\odot} \mathrm{yr}^{-1}$ and $\dot{M}_{\mathrm{HeI}}=7.5 \times 10^{-10} M_{\odot} \mathrm{yr}^{-1}$. Evidence of outflow activity comes from the detection of the [O I] $\lambda 5577$ and [O I] $\lambda 6300$ forbidden lines in an optical spectrum of FU Tau A (Stelzer et al. 2010). All of these facts combined show that the FU Tau system is probably a rarity among BDs, and thus it is of considerable interest to test models describing the formation and evolution of BDs. For this reason we chose to publish the discovery of its molecular outflow separately from the overall presentation of our IRAM survey.

\section{Observations and data reduction}

Observations of the $\mathrm{CO}(2-1)$ line emission were carried out at the IRAM 30 m telescope July 16-18 2011 using the EMIR receivers at $1.3 \mathrm{~mm}$. In a first step, deep integrations were performed towards the protostar and at a reference position located $20^{\prime \prime}$ away. In a second step, in the case of significant variations in the $\mathrm{CO}$ emission between both positions, i.e. beyond the $3 \sigma$ intensity level, more extended mapping at $12^{\prime \prime}$ sampling was performed. The $\mathrm{CO}$ emission map detected toward FU Tau is displayed in Fig. 1.

Observations were carried out in frequency switch mode using a throw of $14.3 \mathrm{MHz}$ at $1.3 \mathrm{~mm}$, with a phase time of $0.2 \mathrm{~s}$. An autocorrelator was used as spectrometer, providing us with a spectral resolution of $40 \mathrm{kHz}$. The weather conditions were rather good and stable, with system temperatures $T_{\text {sys }}$ varying between 200 and $400 \mathrm{~K}$. Each position was observed so as to reach a final rms of about $40 \mathrm{mK}$ per velocity interval of $0.1 \mathrm{~km} \mathrm{~s}^{-1}$, after averaging both polarizations.

Pointing was checked every 1.5 to $2 \mathrm{~h}$ and was found to be very stable, with pointing offset corrections less than $3^{\prime \prime}$. The

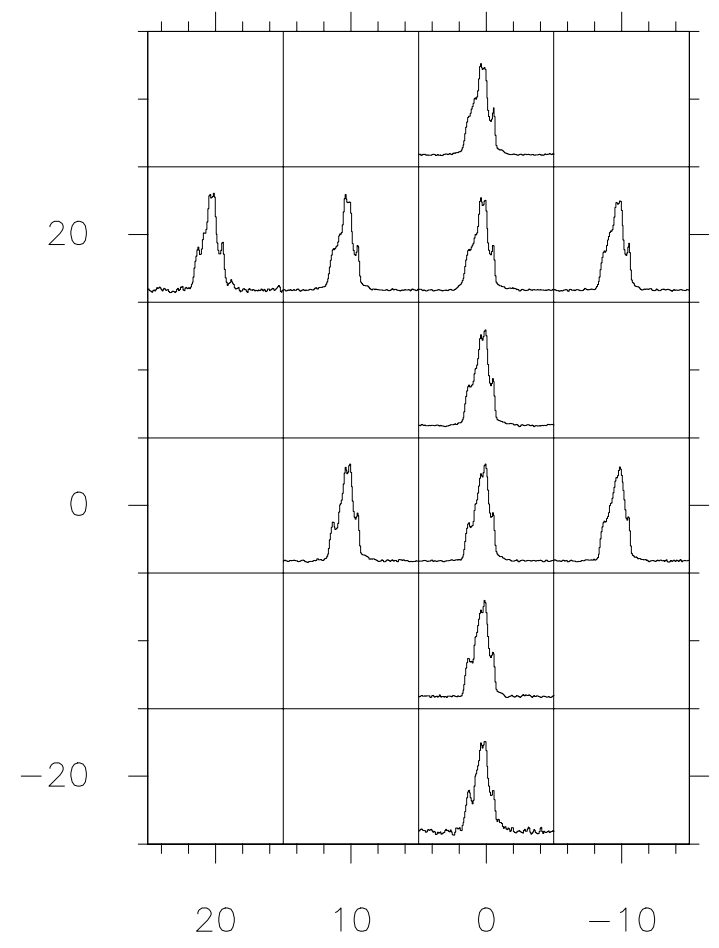

Fig. 1. Map showing the positions of the 11 spectra obtained for FU Tau. The $\mathrm{CO}(2-1)$ line is shown here. The scale of the grid is $0-15 \mathrm{~km} \mathrm{~s}^{-1}$ in $x$ and $-0.6-6 \mathrm{~K}$ in $y$. For all spectra except the $(0,-20)$ and $(+20,+20)$ positions, the rms is less than $40 \mathrm{mK}$. For the $(0,-20)$ and $(+20,+20)$ positions, the noise is $\sim 80 \mathrm{mK}$.

telescope parameters are adopted from the IRAM webpage. At the frequency of the $\mathrm{CO}(2-1)$ line, the main-beam efficiency of the telescope is 0.59 and the half-power beamwidth is $11^{\prime \prime}$. The intensities of the measurements are expressed in units of mainbeam brightness temperature $T_{\mathrm{mb}}$.

The data were reduced using the Continuum and Line Analysis Single-dish Software (CLASS, a GILDAS software ${ }^{1}$ ). In some of the sources, the $\mathrm{CO}$ mesospheric emission line was detected close to the cloud emission, which peaks at $v_{\text {lsr }} \simeq$ $+6 \mathrm{~km} \mathrm{~s}^{-1}$, on the red side of the spectrum. For all our observations we have adjusted a Gaussian to the $\mathrm{CO}$ mesospheric line profile and subtracted it out. The $\mathrm{CO}$ mesopheric line profile is typically a few $\mathrm{K}$ bright, with a linewith of about $1 \mathrm{~km} \mathrm{~s}^{-1}$ (CHECK), much less than the velocity range of the cloud emission and the outflow wing emission. When the outflow feature is on the blueshifted part of the line spectrum, the mesospheric $\mathrm{CO}$ is absolutely harmless. When the observed outflow wing is on the redshifted side, we checked that the $\mathrm{CO}$ mesospheric line is much narrower than the wing velocity range, hence does not hamper the detection of the latter.

\section{Results and discussion}

\subsection{Outflow signature}

In Fig. 2 a magnified view of the wings of the $\mathrm{CO}(2-1)$ emission line at each point on the map of FU Tau (Fig. 1) is shown. The central $(0,0)$ position spectrum is repeated as a dark solid line in all the plots, superimposed on the color lines observed at the other positions. Since the beam is $\approx 11^{\prime \prime}$ wide, it encompasses both FU Tau A and FU Tau B. The bulk of the $\mathrm{CO}(2-1)$ emission comes from the cloud and is centered on the cloud velocity

http://www.iram.fr/IRAMFR/GILDAS/ 

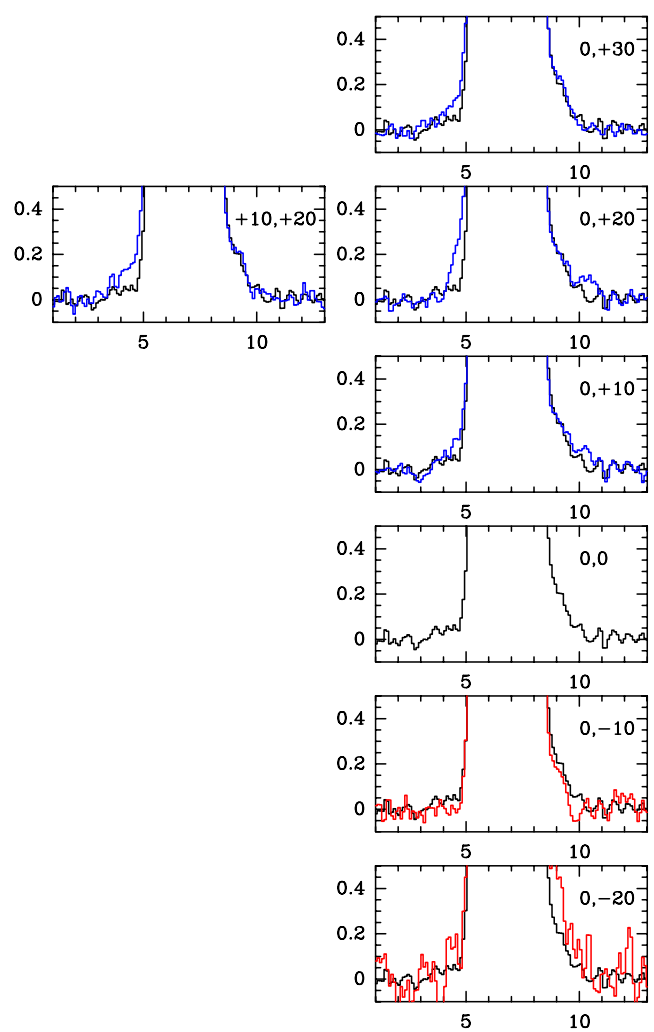

Fig. 2. Montage of some of the $\mathrm{CO}(2-1)$ emission line profiles observed for FU Tau shown with a zoom on the region of the wings. The central $(0,0)$ position spectrum is repeated as a dark solid line in all the plots, superimposed on the lines observed at the other positions, in color. We detect excess emission in the blue-wing between $3 \mathrm{~km} \mathrm{~s}^{-1}$ and $5 \mathrm{~km} \mathrm{~s}^{-1}$, at the $(0,20),(10,20)$ and $(0,30)$ positions. This points to a molecular outflow driven by the FU Tau system.

at $6 \mathrm{~km} \mathrm{~s}^{-1}$. We also search for outflow signature from variations in the $\mathrm{CO}(2-1)$ wing emission with respect to the emission on the central source. The outflow emission that is shifted in velocity with respect to the cloud is much fainter than the cloud emission, and therefore it will be detected in the wings of the $\mathrm{CO}(2-1)$ emission line. Figure 2 shows that a blue component develops in the wing as we move towards the north, with an excess wing emission seen at the $(0,20),(10,20)$ and $(0,30)$ positions between a velocity of $3 \mathrm{~km} \mathrm{~s}^{-1}$ and $5 \mathrm{~km} \mathrm{~s}^{-1}$. There is also a hint of blueshifted excess emission at the $(0,10)$ position, and a red wing appears in the $8-10 \mathrm{~km} \mathrm{~s}^{-1}$ range in the $(0,-20)$ position spectrum. The detection of this excess emission strongly points to a molecular outflow driven by FU Tau. In Fig. 3 we present an integrated intensity map of the blueshifted wing emission in the velocity range $3 \mathrm{~km} \mathrm{~s}^{-1}$ to $6 \mathrm{~km} \mathrm{~s}^{-1}$. This figure shows the positions at which data was collected, and we have superimposed an optical image (WFCAM/UKIRT) of the FU Tau binary taken from Luhman et al. (2009). The detection of the outflow in the form of a blueshifted lobe towards the northeast is clear. Without further data, we estimate a PA of $\sim 20^{\circ}$ for the outflow axis.

\subsection{Outflow parameters}

Following Bachiller et al. (1990), we computed the CO column density in the blue lobe of the outflow with the following equation:

$(\mathrm{CO})_{\left(\mathrm{cm}^{-2}\right)}=1.06 \times 10^{13} T_{\mathrm{mb}} \exp \left(\frac{16.5}{T_{\mathrm{mb}}}\right) \int T_{R}(2-1) \mathrm{d} v$.
Table 1. FU Tau outflow parameters.

\begin{tabular}{ccccc}
\hline \hline $\begin{array}{l}\text { PA } \\
\left({ }^{\circ}\right)\end{array}$ & $\begin{array}{c}\mathrm{i} \\
\left({ }^{\circ}\right)\end{array}$ & $\begin{array}{c}N(\mathrm{CO}) \\
\left(\mathrm{cm}^{-2}\right)\end{array}$ & $\begin{array}{c}M\left(\mathrm{H}_{2}\right) \\
\left(M_{\odot}\right)\end{array}$ & $\begin{array}{c}\dot{M}\left(\mathrm{H}_{2}\right) \\
\left(M_{\odot} / \mathrm{yr}\right)\end{array}$ \\
\hline 20 & 60 & $3.6 \times 10^{16}$ & $4 \pm 1 \times 10^{-6}$ & $5 \pm 1 \times 10^{-10}$ \\
\hline
\end{tabular}

Adopting a gas excitation temperature $T_{\mathrm{mb}} \approx 15 \mathrm{~K}$, with an $\mathrm{H}_{2} / \mathrm{CO}$ ratio of $10^{4}$ and the results of Fig. 3, we computed a mass in the blue lobe of the flow of $M_{B}\left(\mathrm{H}_{2}\right)=4 \pm 0.8 \times 10^{-6} M_{\odot}$.

If we suppose that the momentum of the underlying jet has been transferred entirely to the molecular component that we observe today, we can write

$M_{B}\left(\mathrm{H}_{2}\right)\left\langle V_{\text {max }}\right\rangle=\dot{M}_{\text {jet }}\left\langle V_{\text {jet }}\right\rangle \tau_{\text {dyn }}$.

We measure $\left\langle V_{\max }\right\rangle=3 \mathrm{~km} \mathrm{~s}^{-1}$, and we take a canonical value $\left\langle V_{\text {jet }}\right\rangle=100 \mathrm{~km} \mathrm{~s}^{-1}$; together with $\tau_{\text {dyn }} \approx 200 \mathrm{yr}$ (see Sect. 3.3, first paragraph), we obtain a mass-loss rate for the blueshifted lobe of $\dot{M}_{\text {out }}=6 \pm 1.3 \times 10^{-10} M_{\odot} / \mathrm{yr}$. This value can be modified by various factors. For instance, we can adopt a correction factor to take into account that the jet might have been episodic in the past. Phan-Bao et al. (2011) use a factor of 10 for this purpose. Also, the excitation temperature is uncertain, although the $T_{\mathrm{mb}} \exp \left(16.5 / T_{\mathrm{mb}}\right)$ factor does not vary much over the $T_{21}=10-25 \mathrm{~K}$ range. We could also take extinction effects into account, and the fact that we are only measuring half of the flow emission. Thus this value must be taken as a first-order estimation of the outflow rate and most probably underestimates the rate of the underlying jet. The outflow parameters are summarized in Table 1.

\subsection{Outflow powering source}

Although we cannot disentangle FU Tau A from FU Tau B, it is most likely that the outflow is driven by the primary because forbidden emission associated with the primary has already been detected (Stelzer et al. 2010). Thus for the rest of the discussion we assume that FU Tau A is the driver of the outflow. The peak of the blue lobe is measured at $\sim 20^{\prime \prime}$ from the central source, projected on the plane of the sky. When adopting a projection angle of $60^{\circ}$ (Stelzer et al. 2013), the linear distance is $\approx 50^{\prime \prime}$, corresponding to $7000 \mathrm{AU}$ at the distance of Taurus $(140 \mathrm{pc})$. At $100 \mathrm{~km} \mathrm{~s}^{-1}$, this yields a dynamical age $\tau_{\text {dyn }} \approx 200 \mathrm{yrs}$ for the observed outflow event.

Previous to the results presented here, ISO-Oph 102 and MHO 5, with masses of $60 M_{\text {Jup }}$ and $90 M_{\text {Jup }}$, were the lowest mass objects for which molecular outflows were detected (Phan-Bao et al. 2008, 2011). The outflow mass and mass outflow rate were estimated at $M_{\text {out }}=1.6 \times 10^{-4} M_{\odot}$, and $\dot{M}_{\text {out }}=$ $1.4 \times 10^{-9} M_{\odot} /$ yr for ISO-Oph 102 , and $M_{\text {out }}=7.0 \times 10^{-5} M_{\odot}$, and $\dot{M}_{\text {out }}=9.0 \times 10^{-10} M_{\odot} /$ yr for MHO 5 . Thus our estimates of the mass and mass outflow rate of the FU Tau outflow agree with previous results and are in line with the fact that FU Tau A has the lowest mass of the three objects. The derived values of $\dot{M}_{\text {out }}$ are also consistent with $\dot{M}_{\text {out }}$ measured for the optical components of BD outflows (Whelan et al. 2009).

For ISO-Oph 102, the outflow rate in the molecular component was found to be slightly higher than the optical component. However, it is reasonable that $\dot{M}_{\text {out }}$ for a molecular outflow could be greater than the outflow rate in the underlying jet. Assuming that the jet is powering the molecular outflow (Downes \& Cabrit 2007), the mass outflow rate of the molecular component will grow with time when the jet transfers increasing amounts of energy and momentum. The size of the outflow 


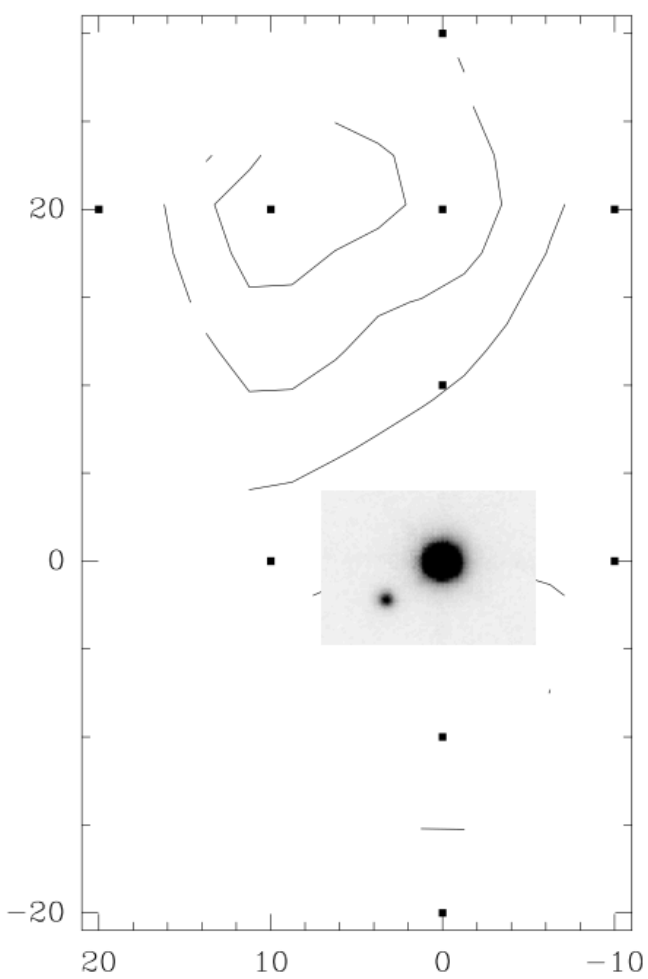

Fig. 3. Integrated map of the blueshifted wing emission in the velocity range $3-5 \mathrm{~km} \mathrm{~s}^{-1}$. The LSR velocity of the BD is $+6 \mathrm{~km} \mathrm{~s}^{-1}$. The black squares mark the positions at which observations we made. The level are $0.1,0.15,0.2$, and $0.25 \mathrm{~K} \mathrm{~km} \mathrm{~s}^{-1}$. Clearly we see a blue outflow lobe at a PA of $\sim 20^{\circ}$. We have superimposed a UKIDSS $K$-band image taken from Luhman et al. (2009) of the BD binary at the same scale and assuming that FU Tau-A is at the central position.

of $\sim 20^{\prime \prime}$ is compatible with the ISO-Oph 102 and MHO 5 flows and with observations of BD optical outflows. The agreement between the scale of the molecular and optical components is important if one is to accept that the jet drives the molecular flow. Finally, we compared the mass outflow rate of the $\mathrm{CO}$ outflow from FU Tau with the derived mass accretion rate and found the ratio of mass outflow to mass accretion, $\dot{M}_{\text {out }} / \dot{M}_{\text {acc }}=0.8$ to 1.7 . $\dot{M}_{\text {out }} / \dot{M}_{\text {acc }}$ measured for other BDs and VLMSs has also been found to be high compared to $\mathrm{T}$ Tauri stars where it is measured at $1-10 \%$ (Bacciotti et al. 2011; Whelan et al. 2009).

We stress that such a high value of $\dot{M}_{\text {out }} / \dot{M}_{\text {acc }}$ cannot be used as a clue that this outflow removes or has removed a large fraction of the central object's mass (as in the Machida et al. 2009 model), because the observed FU Tau molecular outflow results from the entrainment by an underlying jet, and concerns an ejected mass several orders of magnitude lower than in the Machida et al. (2009) model. On this subject, another explanation for the $\dot{M}_{\text {out }} / \dot{M}_{\text {acc }}$ ratio $\approx 1$ in the currently known series of BD sources could be an observational bias, because the first currently available observations are only sensitive to the most extreme jets in brown dwarfs. If this is true, the current ratios should prove much higher than the (as-yet unobserved) mean in BDs. More sensitive observations are thus needed to solve this issue.

\section{Summary}

The discovery of a molecular outflow driven by FU Tau A adds significantly to the interesting properties of this source and its binary companion. The FU Tau binary has a larger separation than most binary systems and is thought to have formed in relative isolation. Both components harbor Class II accretion disks, and FU Tau A is somewhat overluminous for its spectral type. That FU Tau A is driving an outflow demonstrates that, despite having unusual characteristics, it still exhibits properties that are strongly linked to the formation of low-mass protostars. The mass, scale, and mass outflow rate that we measured for the FU Tau A CO outflow agrees with previous observations of $\mathrm{BD}$ molecular outflows. While this result is another important piece of evidence linking the way BDs form to low-mass star formation, the derived ratio of mass outflow to accretion rates is much higher than what is observed in lowmass protostars and, in particular, the T Tauri stars. For other BDs the two rates have been found to be comparable thus these new results for the FU Tau system support other studies of BD outflow activity (Bacciotti et al. 2011; Whelan et al. $2009)$. The $\dot{M}_{\text {out }} / \dot{M}_{\text {acc }}$ ratio $\approx 1$ in the currently known series of BD sources could be due to an observational bias, because the first currently available observations could only be sensitive to the most extreme jets in brown dwarfs. More observations are needed to solve this issue. FU Tau is an excellent candidate for follow-up observations with submillimeter interferometers such as the Plateau de Bure interferometry or the Submillimeter Array (SMA). With higher angular resolution observations, we will be able to fully resolve the outflow, search for a redshifted lobe, and confirm whether FU Tau A is the driving source of the flow.

Acknowledgements. The authors would like to acknowledge the help of the IRAM $30 \mathrm{~m}$ team during the observations. E.T. Whelan is supported by an IRCSET-Marie Curie International Mobility Fellowship in Science, Engineering and Technology within the 7th European Community Framework Programme. We thank the referee, S. Mohanty, for a fast and thorough review that helped to improve the quality of this paper.

\section{References}

Bacciotti, F., Whelan, E. T., Alcalá, J. M., et al. 2011, ApJ, 737, L26

Bachiller, R. 1996, ARA\&A, 34, 111

Bachiller, R., Martin-Pintado, J., Tafalla, M., Cernicharo, J., \& Lazareff, B. 1990, A\&A, 231, 174

Cabrit, S., Raga, A., \& Gueth, F. 1997, in Herbig-Haro Flows and the Birth of Stars, eds. B. Reipurth, \& C. Bertout, IAU Symp., 182, 163

Cabrit, S., Ferreira, J., \& Dougados, C. 2011, in IAU Symp. 275, eds. G. E. Romero, R. A. Sunyaev, \& T. Belloni, 374

Downes, T. P., \& Cabrit, S. 2007, A\&A, 471, 873

Downes, T. P., \& Ray, T. P. 1999, A\&A, 345, 977

Jayawardhana, R., Mohanty, S., \& Basri, G. 2003, ApJ, 592, 282

Jones, B. F., \& Herbig, G. H. 1979, AJ, 84, 1872

Luhman, K. L. 2012, ARA\&A, 50, 65

Luhman, K. L., Mamajek, E. E., Allen, P. R., Muench, A. A., \& Finkbeiner, D. P. 2009, ApJ, 691, 1265

Machida, M. N., Inutsuka, S.-I., \& Matsumoto, T. 2009, ApJ, 699, L157

Monin, J.-L., Guieu, S., Pinte, C., et al. 2010, A\&A, 515, A91

Natta, A., Testi, L., Comerón, F., et al. 2002, A\&A, 393, 597

Natta, A., Testi, L., Muzerolle, J., et al. 2004, A\&A, 424, 603

Padoan, P., \& Nordlund, А. 2004, ApJ, 617, 559

Phan-Bao, N., Riaz, B., Lee, C.-F., et al. 2008, ApJ, 689, L141

Phan-Bao, N., Lee, C.-F., Ho, P. T. P., \& Tang, Y.-W. 2011, ApJ, 735, 14

Reipurth, B., \& Bally, J. 2001, ARA\&A, 39, 403

Ricci, L., Testi, L., Natta, A., Scholz, A., \& de Gregorio-Monsalvo, I. 2012, ApJ, 761, L20

Rigliaco, E., Natta, A., Randich, S., et al. 2011, A\&A, 526, L6

Scholz, A., Stelzer, B., Costigan, G., et al. 2011, MNRAS, 1730

Stelzer, B., Scholz, A., Argiroffi, C., \& Micela, G. 2010, MNRAS, 408, 1095

Stelzer, B., Alcala, J., Scholz, A., et al. 2013, A\&A, in press DOI: $10.1051 / 0004-6361 / 201220736$

Whelan, E. T., Ray, T. P., Bacciotti, F., et al. 2005, Nature, 435, 652

Whelan, E. T., Ray, T. P., Podio, L., Bacciotti, F., \& Randich, S. 2009, ApJ, 706, 1054

Whitworth, A., Bate, M. R., Nordlund, A., Reipurth, B., \& Zinnecker, H. 2006 [arXiv: astro-ph/0602367] 